\title{
Articles
}

\section{Erythromycin improves glycaemic control in patients with Type II diabetes mellitus}

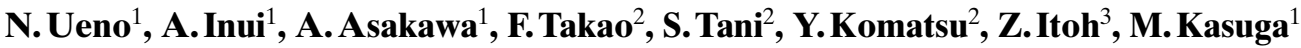 \\ ${ }^{1}$ Second Department of Internal Medicine, Kobe University School of Medicine, Kobe, Japan \\ ${ }^{2}$ Department of Internal Medicine, Komatsu Hospital, Neyagawa, Japan \\ ${ }^{3}$ Gunma University, Professor Emeritus, Maebashi, Japan
}

\section{Abstract}

Aims/hypothesis. Erythromycin mimics the effect of the gastrointestinal hormone motilin by binding to its receptor and acting as a motilin agonist. We recently found that motilin stimulates insulin secretion at lower doses than doses required to stimulate gastric contractile activity. We studied the effects of erythromycin on insulin secretion and glycaemic control in patients with diabetes mellitus.

Methods. Inpatients $(n=34)$ with Type II (non-insulin-dependent) diabetes mellitus were randomly assigned to receive either erythromycin ( $400 \mathrm{mg}$ orally three times a day, $n=19)$ or a placebo $(n=15)$ for 1 week (first study). Another 34 outpatients with Type II diabetes were also treated with erythromycin (200 mg orally three times a day, $n=17$ ) or a placebo $(n=17)$ for 4 weeks (second study). Finally, nine inpatients with Type II diabetes and eight normal control subjects received intravenous erythromycin $\left(10 \mathrm{mg} \cdot \mathrm{kg}^{-1} \cdot \mathrm{h}^{-1}\right)$ or saline infusion and insulin secretion was examined (third study).
Results. Erythromycin lowered fasting blood glucose and fructosamine concentrations $(p<0.01)$ and increased basal as well as glucose-stimulated insulin secretion $(p<0.05-0.01)$ (first study). Low doses of erythromycin treatment for 4 weeks also significantly improved glycaemic control in Type II diabetic patients (second study). Erythromycin infusion significantly increased plasma insulin and decreased glucose concentrations in Type II diabetic and control subjects and greatly potentiated glucose-induced insulin secretion in the latter (third study).

Conclusion/interpretation. These results indicate that erythromycin given orally has an antidiabetogenic effect and therefore erythromycin derivatives that lack the antibacterial activity could have a therapeutic value in Type II diabetic patients. [Diabetologia (2000) 43: 411-415]

Keywords Erythromycin, motilin, insulin, Type II diabetes mellitus, motilide.
Erythromycin, one of the most common macrolide antibiotics, has long been known to have side effects on the gastrointestinal tract, especially when it is introduced rapidly into the vein $[1,2]$. Erythromycin and its derivatives are now called motilides because

Received: 25 October 1999 and in revised form: 10 December 1999

Corresponding author: A. Inui, $\mathrm{MD}, \mathrm{PhD}$, Second Department of Internal Medicine, Kobe University School of Medicine, 7-5-2 Kusunoki-cho, Chuo-ku, Kobe 650-0017, Japan Abbreviations: EM, Erythromycin. they are specific agonists of motilin receptors, thereby mimicking motilin's stimulatory actions on gastrointestinal motility $[1,2]$. Erythromycin has been successfully shown to accelerate delayed gastric emptying associated with various conditions such as diabetes mellitus, cancer therapy, postvagotomy, and progressive systemic sclerosis $[1,2]$. In vivo studies in humans with the motilin agonist erythromycin have suggested that, at low concentrations, the effect of erythromycin is neurally mediated but the response at high concentrations reflects a direct muscular effect [3].

Recently we found that motilin's actions are not limited to gastrointestinal motility [4-6]. Physiologi- 
Table 1. Clinical characteristics and effects of erythromycin $(1200 \mathrm{mg} /$ day for 1 week $)$ or a placebo on glycaemic control in inpatients with Type II diabetes

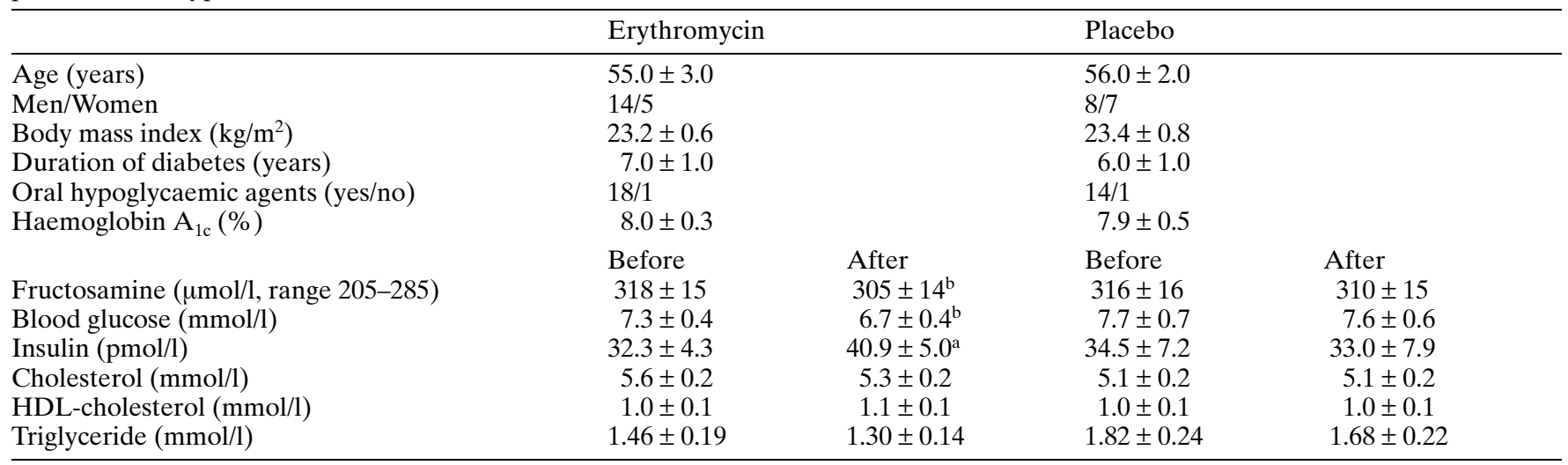

${ }^{\mathrm{a}} p<0.05,{ }^{\mathrm{b}} p<0.01$ vs pretreatment values

Table 2. Clinical characteristics and effects of erythromycin (600 mg/day for 4 weeks) or a placebo on glycaemic control in outpatients with Type II diabetes

\begin{tabular}{|c|c|c|c|c|c|c|}
\hline & \multicolumn{3}{|c|}{ Erythromycin } & \multicolumn{3}{|l|}{ Placebo } \\
\hline Age (years) & $55.0 \pm 4.0$ & & & $55.0 \pm 3.0$ & & \\
\hline Men/Women & $7 / 10$ & & & $8 / 9$ & & \\
\hline Duration of diabetes (years) & $7.0 \pm 1.0$ & & & $6.0 \pm 1.0$ & & \\
\hline Oral hypoglycaemic agents (yes/no) & Before & 4 weeks after & $\begin{array}{l}8 \text { weeks after } \\
\text { (Wash-out) }\end{array}$ & Before & 4 weeks after & $\begin{array}{l}8 \text { weeks after } \\
\text { (Wash-out) }\end{array}$ \\
\hline Body mass index $\left(\mathrm{kg} / \mathrm{m}^{2}\right)$ & $23.9 \pm 0.7$ & $23.7 \pm 0.7$ & $23.7 \pm 0.7$ & $23.7 \pm 0.6$ & $23.6 \pm 0.7$ & $23.6 \pm 0.6$ \\
\hline Insulin (pmol/l) & $27.3 \pm 4.3$ & $38.1 \pm 6.5$ & - & - & - & - \\
\hline Pancreatic glucagon (ng/l) & $120 \pm 12$ & $115 \pm 10$ & - & - & - & - \\
\hline
\end{tabular}

${ }^{\mathrm{a}} p<0.05,{ }^{\mathrm{b}} p<0.01$ vs pretreatment values

cal doses of exogenous motilin stimulates cyclic release of insulin and pancreatic polypeptides from the pancreas through the vagal-cholinergic muscarinic pathway in dogs $[4,5]$. The insulin secretagogue action of motilin, irrespective of its action on gastrointestinal motor activity [5], prompted us to investigate whether or not erythromycin also stimulates insulin secretion and improves glycaemic control in patients with Type II (non-insulin-dependent) diabetes mellitus.

\section{Subjects and methods}

Patients. We studied 34 inpatients with Type II diabetes who were randomly assigned to receive either erythromycin (400 mg of erythromycin stearate [Dainabot (Tokyo, Japan), orally three times a day, $n=19$ ] or a placebo $(n=15)$ for 1 week (first study, Table 1). They were admitted to hospital at least 2 weeks before the study and received a standard diet. Another 34 patients with Type II diabetes were treated with erythromycin (200 mg orally three times a day, $n=17)$ or a placebo $(n=17)$ for 4 weeks at the outpatient clinic (second study, Table 2). All patients were being treated with either diet therapy only or diet therapy and sulphonylureas [glibenclamide
(2.5-5 mg/day) or gliclazide (40-80 mg/day)] but not with insulin, which continued during the study. They were instructed to follow a standard diet for at least 2 weeks before the study and during the study. Finally nine inpatients with Type II diabetes and eight normal control subjects received intravenous erythromycin [erythromycin lactobionate (Dainabot), $10 \mathrm{mg} \cdot \mathrm{kg}^{-1} \cdot \mathrm{h}^{-1}$ ] or saline infusion for $60 \mathrm{~min}$, with control subjects being examined with $(n=6)$ or without $(n=8)$ intravenous glucose infusion $(37 \mathrm{~g} / 500 \mathrm{ml})$ (third study). None of the patients had impaired renal function, autonomic neuropathy or proliferative retinopathy and all had normal liver function. All patients and control subjects gave informed consent to participate in the study, which had been approved by Komatsu Hospital's ethics committee.

Methods. Blood samples of fasting subjects were taken using an indwelling venous catheter before, during and after the treatment for measurement of blood glucose, fructosamine and haemoglobin $\mathrm{A}_{1 \mathrm{c}}$ as well as insulin and glucose responses to an intravenous glucose loading $(0.2 \mathrm{~g} / \mathrm{kg}$ body weight; first study) which was done before and on the last day of the treatment. Insulin and glucose responses to intravenous erythromycin infusion were also measured in the third study. Blood was collected in tubes containing $1.2 \mathrm{mg}$ EDTA and $400 \mathrm{kU}$ aprotinin (Trasylor, Bayer, Leverkusen, Germany). Plasma obtained by centrifugation was stored at $-40^{\circ} \mathrm{C}$ until assayed. Blood glucose was determined by the glucose oxidase method, fruc- 

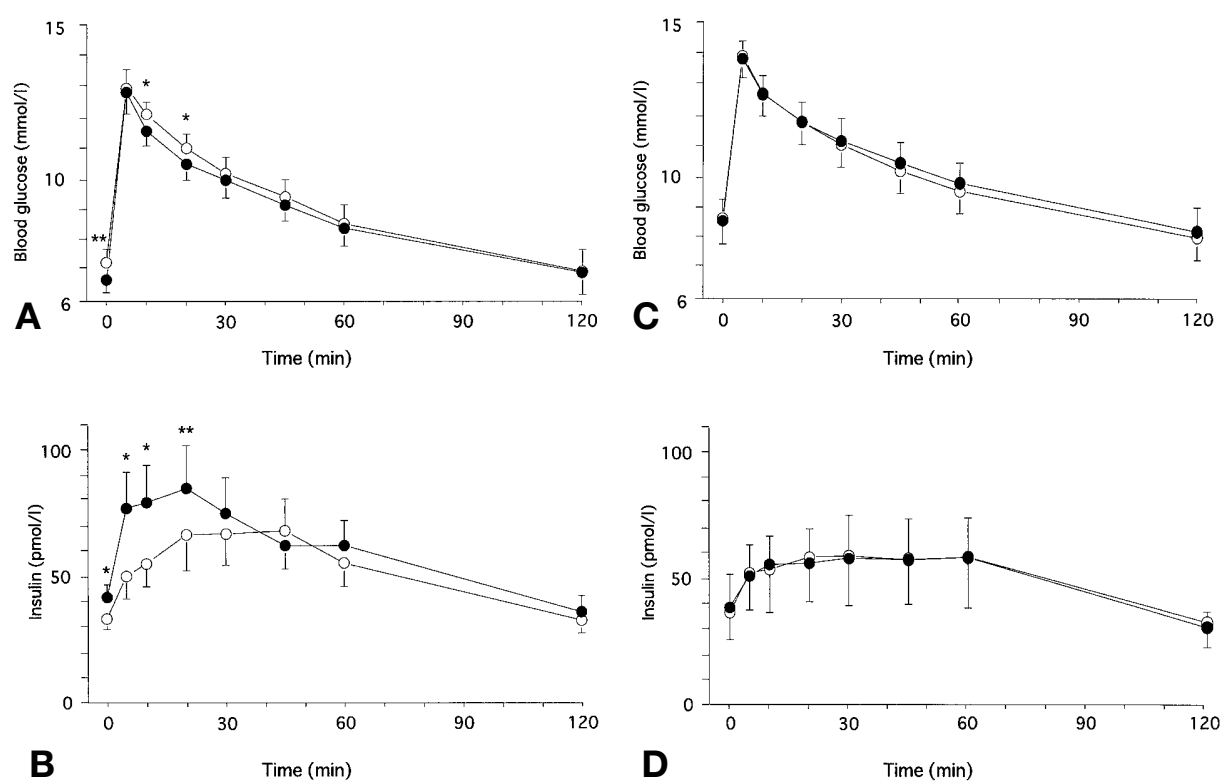

Fig. 1A-D. Effect of erythromycin treatment (1200 mg . day $^{-1}$. week, $\left.n=19\right)$ on blood glucose $(\mathbf{A})$ or insulin $(\mathbf{B})$ or a placebo $(n=15)(\mathbf{C}, \mathbf{D})$ on blood glucose and insulin responses to intravenous glucose loading $(0.2 \mathrm{~g} / \mathrm{kg}$ body weight $)$ in Type II diabetic patients. Glucose tolerance test was done before the treatment $(\bigcirc-\bigcirc)$ and on the last day of the treatment (O-O). Orally given erythromycin (but not the placebo) augmented the initial insulin secretion and lowered blood glucose responses. $* p<0.05, * * p<0.01$ vs control subjects

tosamine by the nitroblue tetrazolium colorimetric test (NBT) method, and haemoglobin $\mathrm{A}_{1 \mathrm{c}}$ by the HPLC method. Plasma insulin was measured with a commercially available kit, Insulin-III (Boehringer-Mannheim, Mannheim, Germany) [7]. Pancreatic glucagon was measured using a previously described assay [8].

Statistical analysis. Results are expressed as means \pm SEM. Testing for significant differences was carried out with analysis of variance (StatView, version 4.5, Abacus Concepts, Berkeley, Calif., USA) followed by the $t$ tests. The responses of blood glucose and insulin were calculated as the integrated area under the curve for 120 min after intravenous glucose injection [9]. A $p$ value lower than 0.05 was considered significant.

\section{Results}

There were no background differences between the treatment and the placebo groups of Type II diabetic patients (first and second studies), including age, gender, body mass index, oral hypoglycaemic treatment, fasting blood glucose, and haemoglobin $A_{1 c}$ values (Tables 1,2). Treatment for 1 week with oral erythromycin (1200 mg per day) reduced fructosamine concentrations in diabetic patients $(p<0.01$, first study, Table 1). Decreases in fasting glucose concentrations were evident after the second day of erythromycin treatment and were maintained throughout; blood

glucose increased, however, as soon as erythromycin was discontinued [blood glucose was $7.2 \pm 0.4$ (before the treatment), $6.5 \pm 0.5$ (third day of the treatment) and $7.3 \pm 0.4 \mathrm{mmol} / \mathrm{l}$ (second day after the treatment)]. Erythromycin increased basal insulin secretion as well as secretion induced by intravenous glucose loading for 5 to $20 \mathrm{~min}$ ( $p<0.05-0.01$, Fig. 1$)$. Total integrated area under the plasma insulin curve for $120 \mathrm{~min}$ was $3622.31 \pm 620.57 \mathrm{pmol} \cdot \mathrm{min}^{-1} \cdot \mathrm{l}^{-1}$ and $4199.43 \pm 705.43 \mathrm{pmol} \cdot \mathrm{min}^{-1} \cdot \mathrm{1}^{-1}$ before and after treatment, respectively $(p<0.05)$. Blood glucose concentrations were lower at $0 \mathrm{~min}(p<0.05$, basal $)$, 10 and $20 \mathrm{~min}$ after the treatment $(p<0.05-0.01)$ and total integrated area under the blood glucose curve decreased $\left(569.48 \pm 29.85 \mathrm{mmol} \cdot \mathrm{min}^{-1} \cdot \mathrm{l}^{-1}\right.$ vs $\left.553.24 \pm 29.98 \mathrm{mmol} \cdot \min ^{-1} \cdot 1^{-1}, \quad p<0.05\right)$. Neither insulin $\quad\left(4461.22 \pm 1047.56 \mathrm{pmol} \cdot \mathrm{min}^{-1} \cdot \mathrm{l}^{-1} \quad \mathrm{vs}\right.$ $\left.4337.94 \pm 879.41 \mathrm{pmol} \cdot \mathrm{min}^{-1} \cdot \mathrm{l}^{-1}\right)$ nor glucose $\left(583.64 \pm 48.40 \mathrm{mmol} \cdot \mathrm{min}^{-1} \cdot \mathrm{l}^{-1}\right.$ vs $601.65 \pm 40.03$ $\left.\mathrm{mmol} \cdot \min ^{-1} \cdot \mathrm{l}^{-1}\right)$ responses were affected after treatment with the placebo (Fig. 1). Erythromycin had no statistically significant effect on the concentrations of total cholesterol, HDL-cholesterol, or triglyceride (Table 1).

Glycaemic control was also improved in the patients after 4 weeks of treatment with oral erythromycin (600 mg per day, second study, Table 2). Significant changes were observed in fasting blood glucose $(10.33 \pm 0.78$ vs $8.94 \pm 0.44, p<0.05)$ and haemoglobin $\mathrm{A}_{1 \mathrm{c}}$ concentrations $(8.5 \pm 0.2$ vs $7.6 \pm 0.2$, $p<0.01)$. After washing the drug out, haemoglobin $\mathrm{A}_{1 \mathrm{c}}$ increased to the pretreatment value. Placebo treatment had no effect on these variables (Table 2).

Intravenous erythromycin stimulated insulin secretion and decreased blood glucose concentrations in both diabetic patients and healthy volunteers (Fig. 2); the response was, however, more profound in diabetic patients. When blood glucose concentra- 

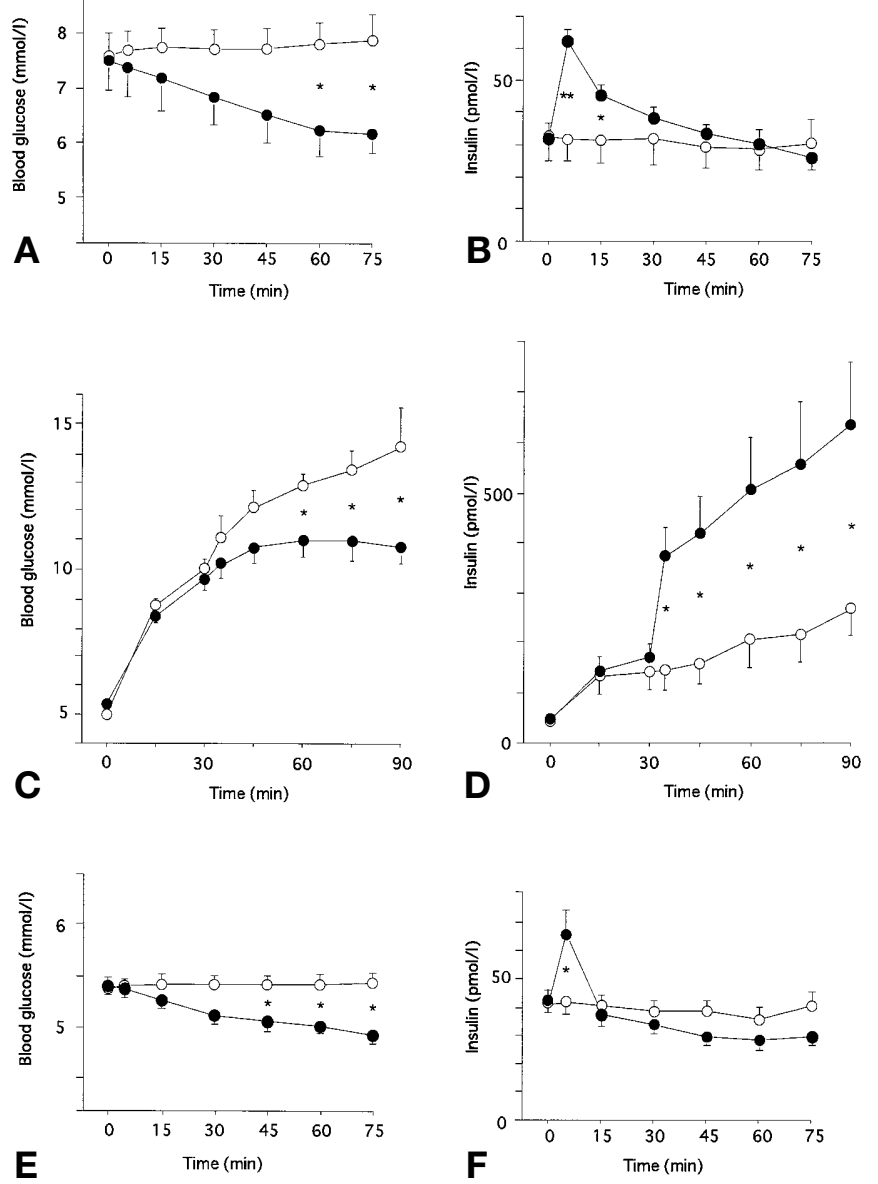

Fig. 2 A-F. Effect of intravenous erythromycin infusion $\left(10 \mathrm{mg} \cdot \mathrm{kg}^{-1}\right.$ body weight $\left.{ }^{-1} \cdot \mathrm{h}^{-1}\right)$ in Type II diabetic patients $(n=9)$ on blood glucose $(\mathbf{A})$ and insulin (B) concentrations and on blood glucose $(\mathbf{C})$ and insulin $(\mathbf{D})$ concentrations of normal control subjects, examined with $(n=6)$ or on blood glucose $(\mathbf{E})$ and insulin $(\mathbf{F})$ concentrations of normal control subjects examined without $(n=8)$ intravenous glucose infusion $(37 \mathrm{~g} / 500 \mathrm{ml})$. Intravenous erythromycin (but not saline) stimulated basal insulin secretion and lowered blood glucose concentrations in diabetic patients (A) and normal control subjects $(\mathbf{E}, \mathbf{F})$. Erythromycin greatly potentiated glucose-induced insulin secretion in control subjects $(\mathbf{C}, \mathbf{D})$. ${ }^{*} p<0.05$, $* * p<0.01$ vs control subjects. $\bigcirc$, saline; $\mathbf{O}$, erythromycin

tions were raised by intravenous glucose infusion in normal control subjects, the effect of erythromycin on insulin secretion was more significant (Fig.2). Erythromycin effected a sustained increase of plasma insulin concentrations which was greater than that in response to glucose alone $(p<0.05)$. Saline given as a control had no statistically significant effect on the concentrations of glucose and insulin and erythromycin had no significant effect on pancreatic glucagon secretion as reported previously $[7,10]$ (not shown).

There were no side effects of oral erythromycin treatment such as decreased appetite and diarrhea, except in two outpatients who had reported hypoglycaemia occurring 1 or 4 weeks after the start of erythromycin treatment. No changes in the results of liver- function tests or other laboratory tests were observed during the 1-week or 4-week treatment period.

\section{Discussion}

We showed that erythromycin given orally for 1 week or 4 weeks improves glycaemic control in patients with Type II diabetes, at lower doses (600mg$1200 \mathrm{mg}$ a day) than those used for the antibacterial activity of the compound. Erythromycin enhances insulin release, both basal and glucose-stimulated in particular, confirming our previous observations in dogs [5] and mice (unpublished data) that motilin and motilides stimulate the beta cells.

In dogs, the insulin release induced by motilin or EM523, a non-peptide motilin agonist of erythromycin derivative, is abolished by pretreatment with atropine and a 5-hydroxytryptamine-3 receptor antagonist and by truncal vagotomy $[5,10]$. Because there are no motilin receptors in the pancreas [1], the actions of motilin and motilides on insulin secretion are most likely to be mediated by vagal-cholinergic muscarinic pathways linking to serotonergic receptors, a common mechanism in the stimulatory effect of motilin on muscle contraction in the stomach and on pancreatic polypeptide secretion from the endocrine pancreas $[1,4,10]$. In this study, the enhancement of basal as well as intravenous glucose-stimulated insulin secretion by erythromycin was especially evident in the first 20-30 min, suggesting that erythromycin evokes neurally-mediated initial insulin secretion, a characteristic defect observed in Type II diabetic patients [11, 12]. A very recent report on identification of motilin receptor in the human enteric nervous system could support the idea [13]. It is well known that early insulin secretion is very important for maintaining prandial glucose within limits, preventing enhanced blood glucose and thus increased (but delayed) insulin response which might lead to diabetic complications [12].

Our study does not fully address the therapeutic potential of erythromycin or its derivatives for glycaemic control. The observation period is limited to 4 weeks due to concern about the potential for the emergence of resistent bacterial strains during longterm use [14], which could prevent valid conclusions about the effects of oral treatment with erythromycin on the long-term control of diabetes. It is also not apparent from our study which oral doses are the best candidates for use in clinical trials. The considerable improvement of glycaemic control is, however, the rationale for evaluating the drug's therapeutic potential in the treatment of Type II diabetes. It was previously reported that intravenous erythromycin greatly improved impaired gastric emptying in ten patients with severe diabetic gastroparesis and the drug was active when given orally for 4 weeks at a dosage of 
$750 \mathrm{mg}$ a day [15]. Similar findings were reported in a group of 14 patients with idiopathic or diabetic gastroparesis, 5 of whom maintained symptomatic improvements for an average of 8.4 months, at a dosage of $1000 \mathrm{mg}$ a day [16]. These results indicate that erythromycin is effective both as a gastroprokinetic and an antidiabetogenic agent at similar oral doses without producing apparent desensitization. Because as many as $30 \%$ of diabetic patients were reported to have apparent gastric motor disorders, such as early satiety, abdominal distention, nausea, vomiting and anorexia [1], erythromycin derivatives that lack the antibacterial activity could be of therapeutic value in diabetic patients, particularly those having gastrointestinal motility disorders $[7,16]$.

Our preliminary study indicates that oral treatment with erythromycin increases the beta-cell secretory capacity through motilin receptors and improves the glycaemic control in Type II diabetic patients, most of whom were treated with currently available oral hypoglycaemic agents. Erythromycin seems to be able to stimulate glucose-dependent as well as vagally-mediated insulin secretion, common defects in Type II diabetes. A number of erythromycin derivatives have been identified as potent motilin agonists that have oral bioavailability, such as ABT-229 and EM574 [1, 2, 17]. Although they are now in phase II clinical trials as a new type of gastroprokinetics, the potential role of these motilides as a new antidiabetogenic agent has never been examined and studies are much needed.

Acknowledgements. This work was supported in part by Grants-in-Aid for Scientific Research (A) 08559012 and (C) 09671057 from the Ministry of Education, Science, Sports and Culture of Japan (to A. Inui).

\section{References}

1. Itoh Z (1997) Motilin and clinical application. Peptides 18: 593-608

2. Peeters TL (1993) Erythromycin and other macrolides as prokinetic agents. Gastroenterology 105: 1886-1899
3. Coulie B, Tack J, Peeters, T Janssens J (1998) Involvement of two different pathways in the motor effects of erythromycin on the gastric antrum in humans. Gut 43: 395-400

4. Mochiki E, Inui A, Satoh M, Mizumoto A, Itoh Z (1997) Motilin is a biosignal controlling the cyclic release of pancreatic polypeptide via the vagus in fasted dogs. Am J Physiol 272: G224-G232

5. Suzuki H, Mochiki E, Haga N, Satoh M, Mizumoto A, Itoh Z (1998) Motilin controls cyclic release of insulin through vagal cholinergic muscarinic pathways in fasted dogs. Am J Physiol 274: G87-G95

6. Asakawa A, Inui A, Momose K, Ueno N, Fujino MA, Kasuga M (1998) Motilin increases food intake in mice. Peptides 19: 987-990

7. Okano H, Inui A, Ueno N et al. (1996) EM523L, a nonpeptide motilin agonist, stimulates gastric emptying and pancreatic polypeptide secretion. Peptides 17: 985-900

8. Nishino T, Kodaira T, Shin S et al. (1981) Glucagon radioimmunoassay with use of antiserum to glucagon C-terminal fragment. Clin Chem 27: 1690-1697

9. Fujimoto S, Inui A, Kiyota N et al. (1997) Increased cholecystokinin and pancreatic polypeptide responses to a fatrich meal in patients with restrictive but not bulimic anorexia nervosa. Biol Psychiatry 41: 1068-1070

10. Shiba Y, Mizumoto A, Satoh M, Inui A, Itoh Z, Omura S (1996) Effect of nonpeptide motilin agonist EM523 on release of gut and pancreatic hormones in conscious dogs. Gastroenterology 110: 241-250

11. Porte D Jr (1991) $\beta$ cells in type II diabetes mellitus. Diabetes 40: $166-180$

12. Strubbe JH, Steffens AB (1993) Neural control of insulin secretion. Horm Metab Res 25: 507-512

13. Feighner SD, Tan CP, McKee KK et al. (1999) Receptor for motilin identified in the human gastrointestinal system. Science 284: 2184-2188

14. Tannis AA, Baggen MG, Wiggers RH, Ouwendijk RJ (1993) Side-effects of oral erythromycin for treatment of diabetic gastroparesis. Lancet 342: 1431

15. Janssens J, Peeters TL, Vantrappen G et al. (1990) Improvement of gastric emptying in diabetic gastroparesis by erythromycin. N Eng J Med 322: 1028-1031

16. Richards RD, Davenport K, McCallum RW (1993) The treatment of idiopathic and diabetic gastroparesis with acute intravenous and chronic oral erythromycin. Am J Gastroenterol 88: 203-207

17. Choi MG, Camilleri M, Burton DD, Johnson S, Edmonds A (1998) Dose-related effects of N-Demethyl-N-Isopropyl-8,9-anhydroerythromycin A 6,9-hemiacetal on gastric emptying of solids in healthy human volunteers. J Pharmacol Exp Ther 285: 37-40 